Tobias Kleffel*, Philipp Frey, Marion Merklein and Dietmar Drummer

\title{
Analysis of the formation of gap-based leakages in polymer-metal electronic systems with labyrinth seals
}

https://doi.org/10.1515/polyeng-2019-0098

Received March 26, 2019; accepted April 7, 2019; previously published online May 21, 2019

\begin{abstract}
A major challenge in manufacturing polymermetal electronic systems, for example, encapsulations of metal inserts using assembly injection molding, is to create a tight sealing in order to prevent leakage. One typical reason for leakage is the formation of gaps between polymer and metal. Through labyrinth seals, which can be manufactured by structuring of the metal surface, the gapbased leakage networks can be interrupted. However, in order to use the appropriate structure in the right position, knowledge about the formation of the gaps is essential. The research covered in this paper focuses on the analysis of the formation of gap-based leakages in polymer-metal electronic systems with labyrinth seals to improve the component design of such parts. The effects of different embossed labyrinth seals, which differ in depth and features, and an electrochemically manufactured areal seal on the tightness and leakage position are investigated. Furthermore, the formation of the leakage network is presented for the investigated variants.
\end{abstract}

Keywords: embossing; joints; multi-material systems; surface treatments; tightness.

\section{Introduction}

Because of the increasing electrification in all areas of life, for example, transportation, polymer-metal electronic systems with high resilience gain importance [1]. In these systems, the polymer is usually used for the media

*Corresponding author: Tobias Kleffel, Institute of Polymer Technology, Friedrich-Alexander-Universität Erlangen-Nürnberg, Am Weichselgarten 9, 91058 Erlangen, Germany, e-mail: kleffel@lkt.uni-erlangen.de

Philipp Frey and Marion Merklein: Institute of Manufacturing Technology, Friedrich-Alexander-Universität Erlangen-Nürnberg, Egerlandstraße 13, 91058 Erlangen, Germany

Dietmar Drummer: Institute of Polymer Technology, FriedrichAlexander-Universität Erlangen-Nürnberg, Am Weichselgarten 9, 91058 Erlangen, Germany tight enclosure of the electronic, and the metal is usually used for contacting the electronic component through the polymer enclosure. In the automotive area, for example, typical applications are sensors and control units, which have to withstand, beside others, high temperature fluctuations or aggressive media like engine oil [2].

The versatile and flexible assembly injection molding, sometimes also referred to as overmolding, multicomponent injection molding or in-mold assembly, is the common manufacturing method for the mass production of polymer-metal hybrid structures. In this process, preformed metal inserts are placed into the open injection mold and are overmolded by injection of the polymer into the cavity of the closed mold, which results from the combination of the mold walls and the metal inserts [3].

However, because of the lack of compatibility of polymers and metals, failure in the bond can enable the formation of leakages, which can lead to a damage or destruction of the electronic function of the relevant part [4]. The two main failures are cracks in the polymer, which are mainly located at the edges of the metal insert, and gaps between the polymer and the metal insert [5]. The causes for the formation of these failures are mainly thermomechanical stresses, shrinkage, inadequate bonding between the materials and an unfavorable design of the polymer-metal electronic system [6].

Independent from the used materials, there are two main strategies to reduce or avoid the formation of leakages, which can also be combined: the optimization of the bond between the used materials and the optimization of the metal insert geometry, including macroscopic surface structures.

The optimization of the bond is a research area, which is mainly investigated for structural applications. Considering that also single and small leakage paths could lead to an ingress of a medium [7], not all methods that enable a good bond between polymers and metals can also enable a tight bond. Thus, investigations that deal with the tightness of polymer-metal electronic systems focus on such methods that enable full-faced bonding or closed micro-leakage barriers.

One common and approved method for a full-faced bonding is the use of adhesive promoters, which enable a 
firm bonding between polymer and metal [8]. The suitability of different adhesive promoters for tight bonds is shown in several investigations. The use of adhesive promoters, which are based for example on polyamide or silane, is investigated in [9] for a polymer-metal hybrid connector and shows a significant improvement of the tightness compared to an untreated reference. Furthermore, in [10] and [11], a two-stage curing acrylic adhesive used as an adhesive promoter was investigated for a complex enclosure frame, showing a comparable improvement of the tightness. Also, the use of an organosilicon compound as an adhesive promoter is suitable for the manufacturing of tight and strong polymer-metal hybrid structures, which is shown in [12], but lacks detailed information. However, all adhesive promoters share the disadvantage that they have always been adapted to the used materials. Alternatively, microstructures on the surface of the metal insert, which are independent of the used materials, can be used for closed micro-leakage barriers and a full-faced microscale mechanical interlocking according to [13]. The basic requirement for a high tightness [14] and a good bond [15] is the filling of the microstructures with polymer during assembly injection molding. The microstructures can be manufactured by different methods, for example, grinding [16], blasting [17], laser structuring [17] or electrochemical treatments [17], but not all of these methods can manufacture suitable microstructures for tight polymer-metal hybrid structures. For example, grinding [16] or blasting of the metal surface with coarse blasting media [17] leads to an increased bonding strength; however, it promotes leakage. Positive effects on the tightness are investigated for blasting of the metal surface with fine blasting media [17]. According to [17], comparable results can be achieved with microstructures manufactured by laser structuring, which are investigated. Significant improvements on the tightness can be achieved with microstructures manufactured by one-step [18] or two-step [19] electrochemical treatments, which can also reduce sharp edges at the metal inserts. In addition, in [20], it is shown that the electrochemical treatments with hydrochloric acid $(\mathrm{HCl})$ and a combination of $\mathrm{HCl}$ and nitric acid $\left(\mathrm{HNO}_{3}\right)$, which are also used in [18] and [19], can also enhance the bond strength between polymer and metal perpendicular to the joining surface. For the optimization of the metal insert geometry, there are two main approaches: the variation of the edge design for the reduction in the stresses within the polymer and the manufacturing of defined macroscopic surface structures for the creation of labyrinth seals.

It is shown in [21] that cracks within the polymer after temperature cycling can be reduced through micro formed edges of the metal insert. In contrast to metal inserts with sharp edges, no critical increase in the leakage was measured [21]. Thermomechanical finite element simulations of the same variations of the edge design in [22] show that the reason for the reduction in the cracks is a reduction in the stress within the polymer at the rounded edges of the metal insert. The manufacturing of defined macroscopic surface structures on the metal surface also has positive effects on the tightness. It is shown in [16] that structures manufactured by milling and laser structuring result in a significant improvement of the tightness of the used test specimen. Furthermore, a promising approach is the manufacturing of such structures by embossing, which is shown for a polymer-metal hybrid connector in [23]. The main advantage of this method is that the embossing stage can be integrated into a progressive die, the typical production chain for metal inserts. Thus, the structuring process is highly productive, and no separate surface treatments, e.g., adhesive promoters, are required.

Typically, metal inserts are made from sheet metal coils in a stamping press with a progressive die. Within the tool, subsequent steps of cutting, bending and embossing form the final geometry of the metal insert. This is a highly efficient way of producing metal inserts at high volume with up to 2000 strokes per minute. Thus, the integration of an additional embossing step extends the production only slightly. Embossing is a cold forming process where mainly small structures on the surface by indentation of a punch are formed [24]. In contrast to coining, only parts of the material undergo plastification. Since the dimensions of embossed structures are often below $1 \mathrm{~mm}$, embossing can be accounted to the realm of micro forming [25]. Fundamental studies regarding forming structures similar to labyrinth seals, like microchannel structures for micro reactors [26] or coining comparable structures in a multistep micro forming process [27], focus on the aspects of die filling. In contrast to embossing, those investigations are characterized by enhancing the sheet thickness locally, thus requiring high forces. Since embossing of metal inserts is an open forming process, the material flow affects the resulting geometry of the labyrinth seal; thus, a sound knowledge of the forming process and of the formation of gap-based leakages is necessary for the specific design of the embossed structures for tight polymer-metal hybrid parts.

The aim of this paper was the analysis of the formation of gap-based leakages in polymer-metal electronic systems with labyrinth seals. For this purpose, metal inserts with different embossed labyrinth seals, which differ in depth and features, and an areal seal manufactured by an electrochemical treatment are used. The tightness is evaluated by differential pressure and air-under-water tests. Furthermore, the respective leakage paths are shown using microscopic analysis and the air-under-water test. 


\section{Materials and methods}

\subsection{Materials used}

In this research, hot-rolled sheets made of wrought aluminum alloy EN AW-5754 H24 (Hans-Erich Gemmel \& Co. $\mathrm{GmbH}$, Germany, Berlin) were used for the manufacturing of the metal inserts. Good electrical conductivity, low density and high corrosion resistance characterize this aluminum alloy, which are favorable for its usage in resilient polymer-metal electronic systems.

For the electrochemical manufacturing of an areal seal, sodium hydroxide $(\mathrm{NaOH})$ with a concentration of $0.27 \%$ and $\mathrm{HCl}$ with a concentration of $5 \%$ were used. Both reagents were obtained from VWR International GmbH (Germany, Darmstadt).

For the injection molding, commercially available polyamide- 66 granules with 30\% per weight short glass fiber reinforcement (Ultramid A3EG6, BASF SE, Germany, Ludwigshafen), were used, which are commonly used in electronic and automotive applications. Following the manufacturer specifications, the granules were dried at $80^{\circ} \mathrm{C}$ for $12 \mathrm{~h}$ before processing.

\subsection{Test specimen}

In this study, the polymer-metal hybrid connector test specimen in Figure 1 was used for the analysis of the formation of gap-based leakages. The metal insert consists of a head part and a bottom part, which are linked via a rectangular crosspiece, Figure 1B. The crosspiece is the region of interest and resembles a flat connector, which is overmolded. The head and bottom parts are used to position and clamp the test specimen during processing and testing. The front faces of the crosspiece are $5 \mathrm{~mm}$ wide, and the side faces are $1 \mathrm{~mm}$ wide according to the sheet thickness. The area of the polymer component facing the head part will be referred to as the head side, whereas the part facing the bottom will be referred to as the bottom side. In order to investigate the formation of leakage and leakage paths, different variants of metal inserts without (reference $[R]$ ) and with various surface structures for the labyrinth seal were manufactured (Figure 2). In the following, the selection of the used structures is explained.

In order to create simple leakage barriers, inserts with embossed structures across the front faces in the $x y$ plane were overmolded. Two variants with different structure depths were investigated, labeled 0-100 for 0.1-mm-deep and 0-300 for 0.3-mm-deep structures (Figure 2A). Furthermore, the material displacement of the metal during the manufacturing of the structures on the metal insert by embossing was used for the investigation of leakage barriers at the side faces. However, only for a penetration depth of $0.3 \mathrm{~mm}$ (variant 0-300) a significant material displacement in the form of material bulges can be achieved (Figure 2A). A detailed description of the embossing process can be found in the next chapter. In order to realize continuous all-around grooves, variants with additional indentations and different depths were used, which are labeled I0-100 (depth of $0.1 \mathrm{~mm}$ ) and I0-300 (depth of $0.3 \mathrm{~mm}$ ). These variants were chosen to investigate potential effects of the side faces on the overall tightness (Figure 2B). The indentations were manufactured before the embossing
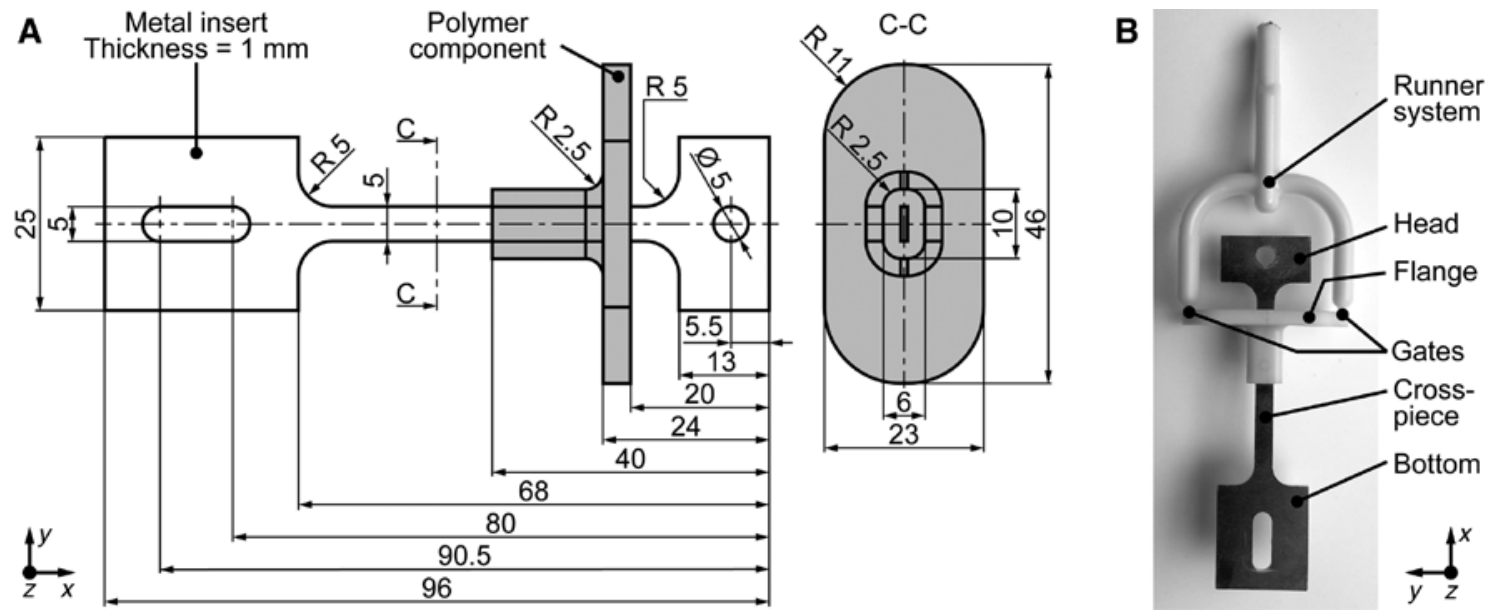

Figure 1: Connector test specimen, referring to [19]. (A) Main dimensions of the metal insert and the polymer component. (B) Connector test specimen with runner system, which allows for the symmetrical filling of the polymer component along the metal insert with two gates at the flange. 
A

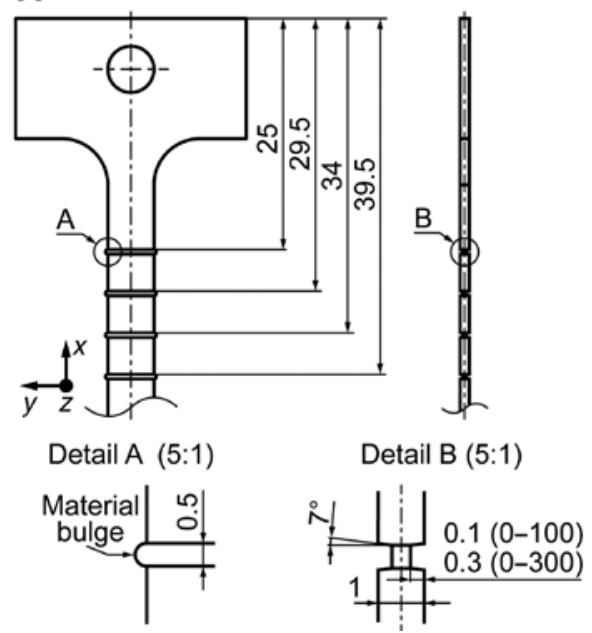

B

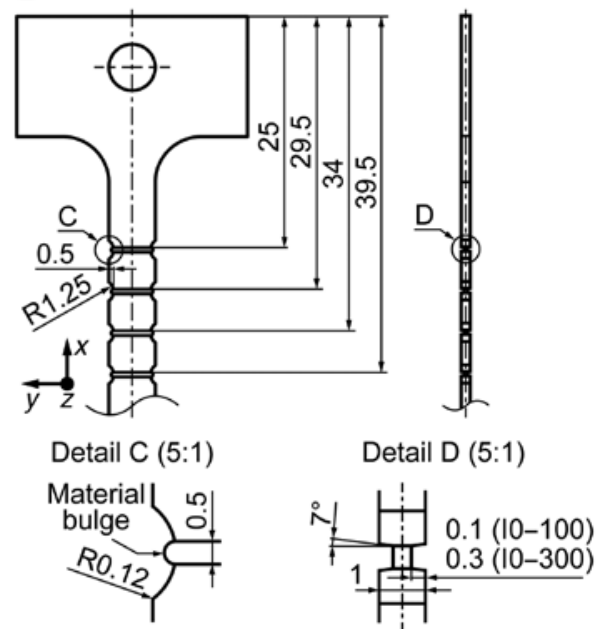

c

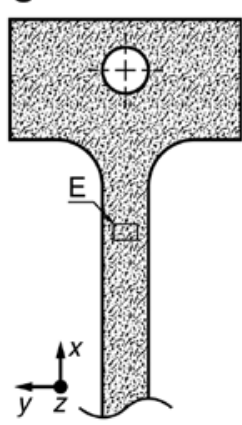

Detail E (1500:1)

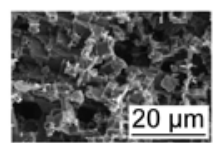

Figure 2: Schematic illustration of the insert variants 0 (A), 10 (B) and MS (C). The schematically marked material bulges of variants $0-300$ (A) and $10-300$ (B) are a result of the structure manufacturing and only formed by a structure depth of $0.3 \mathrm{~mm}$. Detail $E$ shows a scanning electron micrograph (produced with a scanning electron microscope of the type Ultra Plus from Carl Zeiss AG, Germany, Oberkochen) of the structured surface of variant MS, which is representative for all sides of the crosspiece.

process. Similar to the variants described before, only embossed structures with 0.3-mm depth (variant I0-300) lead to a significant formation of material bulges.

In order to achieve an all-over leakage barrier, the crosspiece of variant MS (Figure 2C) has a fully microstructured surface (areal seal). The microstructures feature several undercuts perpendicular to the metal surface, which, if filled with polymer during injection molding, prevent the formation of large gaps according to [18].

\subsubsection{Insert manufacturing}

The metal inserts of all variants were cut from sheets with a laser cutting machine (TruLaser Cell 7020, Trumpf GmbH + Co. KG, Ditzingen, Germany) to ensure smooth surface conditions at the cut surface without work hardening. Afterward, all metal inserts except for the inserts of the reference were structured by embossing or electrochemical treatment to obtain the different variants of labyrinth seals as shown in Figure 2.

\subsubsection{Embossing}

The embossing process includes two identically structured punches, where the lower punch is fixed while the upper punch is moved downward. The metal insert was embossed simultaneously from both sides, while two locating pins (Figure 3) position the insert [23]. The combination of a round and a slotted hole allows for elongation of the metal insert in one direction during the forming

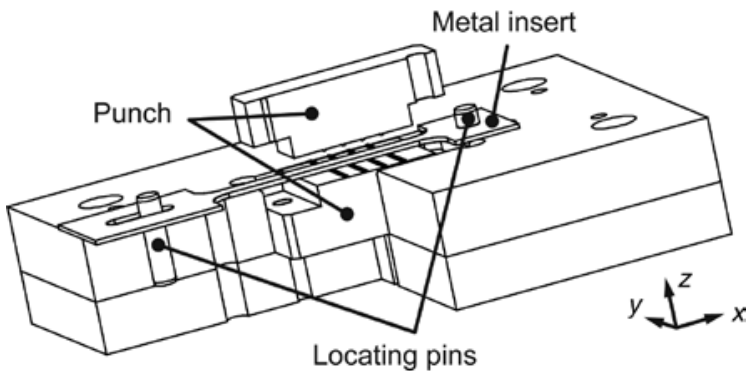

Figure 3: Experimental embossing setup.

process. The embossing was performed with a universal testing machine (RM400, Schenck Trebel GmbH, Germany, Ratingen), with a forming speed of $10 \mathrm{~mm} / \mathrm{min}$. The penetration is stroke controlled and is varied between 0.1 and $0.3 \mathrm{~mm}$ using the same set of punches (Figure 3).

\subsubsection{Electrochemical treatment}

For the electrochemical treatment with $\mathrm{HCl}$ with a concentration of $5 \%$, the same experimental setup as in [22, 23] (Figure 4) was used. The main part of the test setup is an acid temperature-controlling system, which is required to avoid heating of the acid as a result of the exothermic process. It consists of a peristaltic pump (Boxer 9700 Table Top Dispenser, Boxer GmbH, Germany, Ottobeuren), a heat exchanger (custom build, Glasbläserei B+S, Germany, Erlangen) and a cryostat (Lauda Ecoline RE 112, Lauda Dr. R. Wobser GmbH \& Co. KG, Germany, Lauda-Koenigshofen). The cryostat was adjusted to $23^{\circ} \mathrm{C}$ with water as the cooling medium. In addition, a magnetic mixer (RET-G, Janke \& 


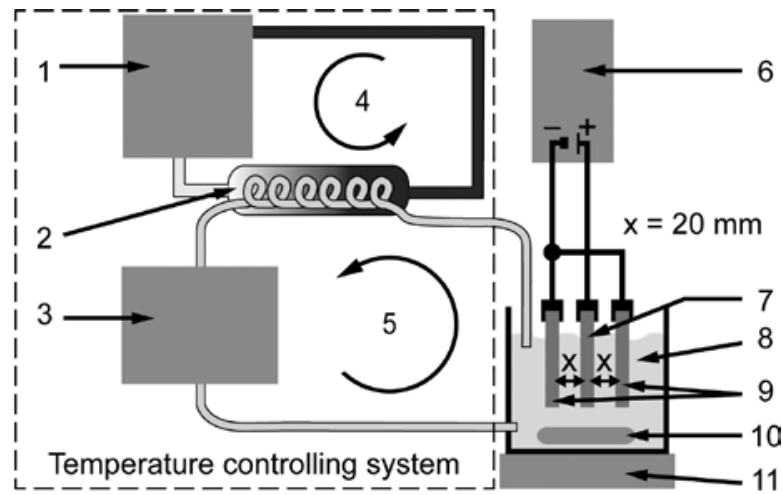

1: Cryostat

2: Heat exchanger

3: Peristaltic pump

4: Secondary cooling circuit

5: Primary cooling circuit

6: Power supply

Figure 4: Experimental setup for the electrochemical treatment of the metal inserts [19].

Kunkel GmbH \& Co. KG, Germany, Staufen) was used to enable circulation of the acid in the laboratory glass. Two metal sheets and a metal insertbetween them wereimmersed in the laboratory glass, which was filled with $800 \mathrm{ml}$ of $\mathrm{HCl}$, and connected to a direct current power supply (Voltcraft DPPS-32-30, Conrad Electronic SE, Germany, Hirschau). The distance between the metal sheets, which were connected as cathodes, and the metal insert, which was connected as the anode, amounted to $20 \mathrm{~mm}$.

In order to obtain the same surface starting conditions at the beginning of the electrochemical treatment, each metal insert was prepared in three steps. First, the metal insert was cleaned with isopropyl alcohol in an ultrasonic bath (Haver USC 200-80, Haver \& Boecker OHG Drahtweberei und Maschinenfabrik, Germany, Oelde) for $1 \mathrm{~min}$, followed by venting until the isopropyl alcohol was vaporized. Following this, the natural oxide layer was removed with a bath in $50^{\circ} \mathrm{C}$ warm $\mathrm{NaOH}$ with a concentration of $0.27 \%$ until an active hydrogen development could be observed (40-60 s). Finally, residues of the $\mathrm{NaOH}$ were removed by rinsing with distilled water.

After the preparation, the metal insert blanks were electrochemically treated in the $\mathrm{HCl}$ solution for $150 \mathrm{~s}$ with a current density of $5 \mathrm{~mA} / \mathrm{mm}^{2}$. Following this, residues of the $\mathrm{HCl}$ solution were removed with distilled water in an ultrasonic bath (Haver USC 200-80, Haver \& Boecker OHG Drahtweberei und Maschinenfabrik, Germany, Oelde) over a period of $60 \mathrm{~s}$. At the end, the microstructured metal inserts were covered in plastic sample bags to prevent damage or contaminations of the microstructures.

\subsubsection{Assembly injection molding}

The test specimens were manufactured reproducibly in a manufacturing cell, which consists of an injection molding machine (Arburg Allrounder 370 U 700-30-30, Arburg GmbH \& Co. KG, Germany, Losburg) and a fully automatic handling system (Wittmann Robot Systeme GmbH, Germany, Nuremberg) including an insert magazine, a pre-heating station and a conveyor. Thus, a constant transfer and cycle time can be ensured. At the beginning of each manufacturing cycle, one metal insert is picked up from the pre-heating station and transferred into the open injection mold by the handling system. Prior to the insertion of the metal insert into the cavity of the injection mold, the manufactured test specimen of the last manufacturing cycle is removed by the handling system. After that, the handling system moves out of the injection mold and places the connector test specimen on the conveyor, which transfers the test specimen into a storage container. Immediately after the handling system has been moved outside of the injection molding machine, the injection mold closes and the next injection cycle starts. In the meantime, a new metal insert is picked up from the insert magazine and placed on the pre-heating station by the handling system. The manufacturing cycle ends with the movement of the handling system to the initial position.

All test specimens were manufactured with the same process parameters. In order to achieve equal cleanliness conditions, all untreated and embossed metal inserts were cleaned with isopropyl alcohol and a cleaning cloth, followed by venting until the isopropyl alcohol was vaporized. The etched metal inserts had no additional cleaning. The pre-heating station was adjusted to $205^{\circ} \mathrm{C}$, which was necessary to ensure that the surface temperature of the crosspiece of the metal insert during the closure of the injection mold was approximately equal to the surface temperature of the injection mold, which was $100^{\circ} \mathrm{C}$. The injection velocity was $80 \mathrm{~mm} / \mathrm{s}$, the melt temperature was $290^{\circ} \mathrm{C}$ and the holding pressure was 250 bar. Immediately after the assembly injection molding, the manufactured test specimen were sealed in plastic foils impervious to water vapor and stored until the investigations started.

\subsection{Test methods}

\subsubsection{Differential pressure test}

Because of its high relevance in industrial serial productions [7], the differential pressure test was used to measure leakage rate as an indicator of tightness. For this purpose, 
the test setup shown in Figure 5A was used, which consists of a leakage test system (Integra DD6, Dr. Wiesner Steuerungstechnik GmbH, Germany, Remshalden) and a suitable clamping device.

The test sequence starts with the clamping of the test specimen into the clamping device, using toggle clamps for reproducible clamping. Afterward, a defined air excess pressure of 1 bar is applied to the reference and the test volume by the compressed air control system. After a period of $2 \mathrm{~s}$, the so-called filling period, the compressed air control system is closed. In the following setting time of $3 \mathrm{~s}$, the compressed air can calm down and adapt to the temperature of the test setup and test specimen. After that, the test and reference volumes are separated from each other by shutting the stop valve, followed by the measurement of the pressure difference between both volumes by a capacitive differential pressure transmitter over a period of $10 \mathrm{~s}$. Finally, the pressure is released over a period of $4 \mathrm{~s}$ by the compressed air control system. In order to correlate the measured pressure differences with leakage rates, which do not depend on the test and reference volume, the test setup was calibrated with a tight reference test specimen and a test leak prior to each measurement series.

Within one measurement series, all test specimens of one metal insert variant were measured once. In total, each test specimen was measured five times because of a possible faulty clamping, which could lead to a measuring error not visible during the test itself. Through the comparison of the five measured leakage rates per test specimen, measuring errors can be identified, so that single measurements can be repeated. For this study, all results have been acquired with correct clamping conditions. For the calculation of the average value and standard deviation of the leakage rate of a variant, the mean value of the repeated measurements of the single test specimens was used. The tests were conducted in standard atmosphere $\left(23^{\circ} \mathrm{C}\right.$ and 50\% humidity) according to DIN EN ISO 291. Immediately after these tests, all tests specimens were once again sealed in plastic foil impervious to water vapor and are stored until the air-under-water test started.

\subsubsection{Air-under-water test}

In order to locate the leakage positions and confirm the test results of the differential pressure test, a modified airunder-water test was used. The used test setup is shown in Figure 5B. At the beginning of the test, a test specimen is clamped in the clamping device while still outside of the measuring basin with two cylinder screws. Afterward, the clamping device with the test specimen is immersed into the measuring basin with distilled water with the longitudinal axis aligned horizontally (Figure 5B). In the next step, the funnel and test tube, which are filled with distilled water, are positioned directly above the end of the polymer component at the bottom side of the test specimen in such a way that the funnel does not capture any bubbles, which could be caused by possible faulty clamping. For the measurement of the leakage rate, a defined air excess pressure of 1 bar is applied to the test volume by the opening of the stop valve over a period of 10 minutes. After the testing time, air bubbles that still stick to the test specimen are detached with a needle and captured by the
A

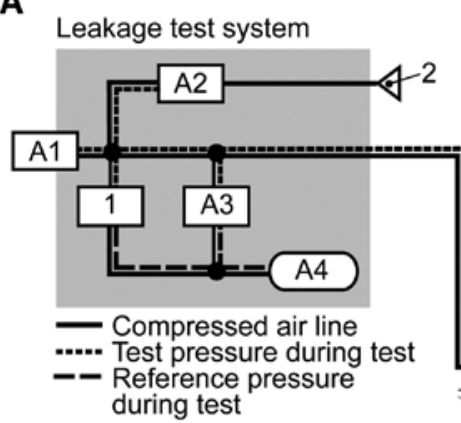

A1: Compressed air coupling for test leak

A2: Compressed air control system

A3: Capacitive differential pressure transmitter

A4: Reference volume

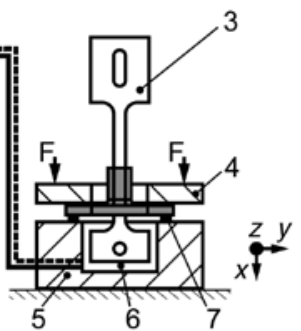

1: Stop valve

2: Compressed air connection

3: Connector test specimen

4: Clamping plate

5: Test block

6: Test volume

7: O-ring

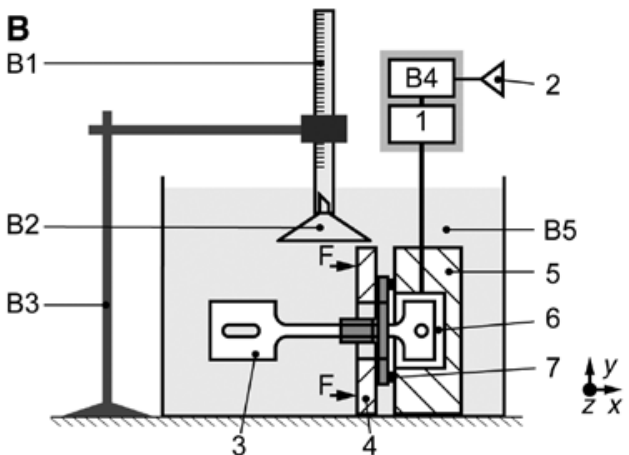

B1: Scaled test tube (water-filled)

B2: Funnel

B3: Tripod

B4: Pressure reduction valve

B5: Tank with water

Figure 5: Test setups for the characterization of the leakage rate. (A) Differential pressure test. (B) Air-under-water test. 
funnel. Then, the air volume $V_{\mathrm{a}}$ leaked during the test can be read off the scaled test tube, and the leakage rate $Q_{\mathrm{L}}$ can be calculated with formula (1), where $t_{\mathrm{t}}$ denotes the testing time.

$$
Q_{\mathrm{L}}=\frac{V_{\mathrm{a}}}{t_{\mathrm{t}}}
$$

After the measurement of the leakage rate, the leakage position can be located. For this purpose, the clamping device with the test specimen is rotated by $90^{\circ}$ so that the longitudinal axis of the test specimen is aligned vertically and the end of the polymer component is properly visible. In the next step, again a defined air excess pressure of 1 bar is applied to the test volume by the opening of the stop valve. Subsequently, the leakage positions can be determined visually by locating the origin of the escaping air bubbles.

Because of the exposure to water, the air-under-water tests were conducted subsequent to the differential pressure test. In contrast to the differential pressure test, each test specimen was measured only once, because single air bubbles in the area of the test block caused by a possible faulty clamping cannot influence the measurement. The tests were conducted at standard atmospheric conditions $\left(23^{\circ} \mathrm{C}\right.$ and $50 \%$ humidity) in accordance with DIN EN ISO 291 [28].

\subsubsection{Microscopy}

In addition to the air-under-water test for the determination of the leakage positions, cross sections of the bottom and head sides in the $x y, x z$ and $y z$ planes (Figure 6) of all the variants were produced to analyze the formation of leakages and the mechanism of action of the used labyrinth seals and the electrochemical manufactured areal seal by microscopy. For this purpose, a microscope (Axio Imager M2m, Carl Zeiss AG, Oberkochen, Germany) was used in combination with the software Imagic IMS from Imagic Bildverarbeitung AG (Switzerland, Glattbrugg) to measure gaps between the polymer component and the metal insert. In $x z$ and $y z$ planes, cross sections of the bottom and head sides of two test specimens per variant were analyzed. Because of difficulty in the preparation of the cross sections in the $x y$ plane in the exact center of the metal insert, only the bottom and head sides of one test specimen per variant were analyzed.

In order to show the influence of gaps between the polymer component and the metal insert on the leakage rate, characteristic gap sizes $L_{1 j}$ and $L_{1}$ were, respectively, calculated using Formula 2 and 3 at the defined measuring position $l_{i j}$, where the index $i$ denotes the side of the metal insert and $j$, if necessary, an additional information about the position. The measuring positions $l_{i j}$ (for example, $\mathrm{a}_{\mathrm{II}}$ ) are defined in Figure 6, where index I denotes the left or upper side, and index II the right or lower side. The additional information $j$ is only used for the characteristic gap sizes in the $y z$ plane, where large areas are analyzed. In total, the 13 characteristic gap sizes $L_{\mathrm{aj}}(j=1,2 \ldots 21), L_{\mathrm{b} j}$ $(j=1,2 \ldots 5), L_{\mathrm{c}}, L_{\mathrm{d}}, L_{\mathrm{e}}, L_{\mathrm{f}}, L_{\mathrm{g}}, L_{\mathrm{h}}, L_{\mathrm{k}}, L_{\mathrm{m}}, L_{\mathrm{n}}, L_{\mathrm{o}}$ and $L_{\mathrm{p}}$ were calculated. The formula used depends on the direction of the shrinkage of the polymer and, thus, on the measuring position. Formula 2 was used for the characteristic gap sizes $L_{\mathrm{aj}}, L_{\mathrm{b}}, L_{\mathrm{d}}, L_{\mathrm{m}}, L_{\mathrm{g}}, L_{\mathrm{n}}$ and $L_{\mathrm{o}}$, where the gaps on both sides are independent from each other and which can be more pronounced on one side than on the other.
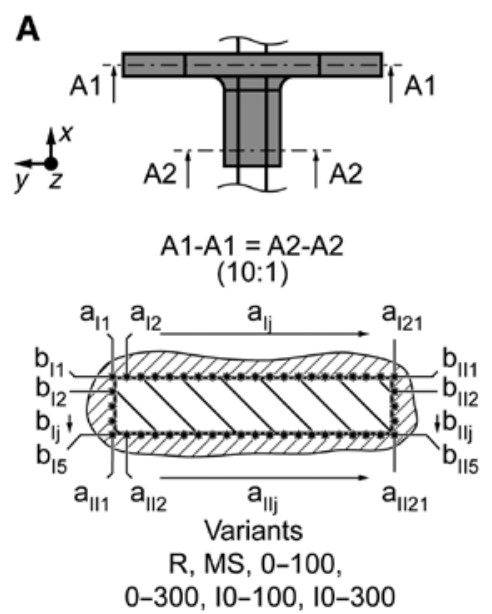

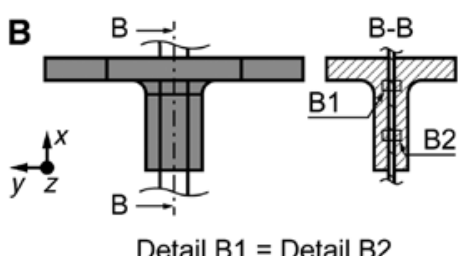
$(20: 1)$

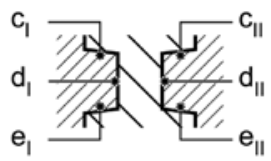

$$
\begin{gathered}
\text { Variants } \\
0-100,0-300 \\
10-100,10-300
\end{gathered}
$$

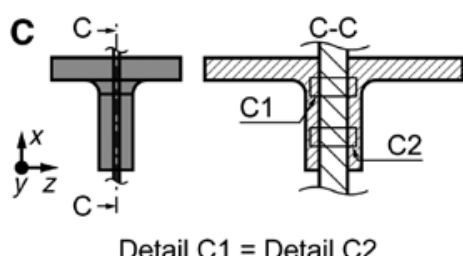

(20:1)
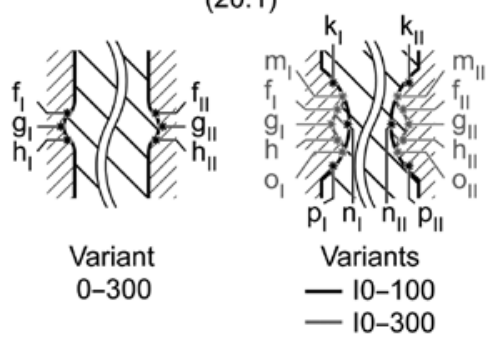

Figure 6: Measuring positions for the calculation of the characteristic gap sizes. (A) $y z$ plane, distance between two points $=250 \mu \mathrm{m}$. (B) $x z$ plane. (C) $x y$ plane. 
Formula 3 was used for the characteristic gap sizes $L_{\mathrm{c}}, L_{\mathrm{e}}$, $L_{\mathrm{f}}, L_{\mathrm{h}}, L_{\mathrm{k}}$ and $L_{\mathrm{p}}$, where the gaps on both sides depend on each other and should theoretically be equal.

$$
\begin{gathered}
L_{\mathrm{lj}}=\sum_{i=1}^{2} l_{i j} \text { or } L_{1}=\sum_{i=1}^{2} l_{i}, \text { respectively } \\
L_{1}=\frac{1}{2} \sum_{i=1}^{2} l_{i}
\end{gathered}
$$

\section{Results and discussion}

\subsection{Tightness}

The leakage rates of the different variants are shown in Figure 7A for both test methods: the differential pressure test and the air-under-water test. It can be seen that variant $\mathrm{R}$ has a high leakage rate of $0.28 \pm 0.29 \mathrm{ml} / \mathrm{min}$, measured with the differential pressure test, which is in the same range compared to previous investigations with the same test setup, materials and almost equal process parameters [18]. The air-under-water test shows a marginally higher average leakage rate of $0.34 \mathrm{ml} / \mathrm{min}$, but with $0.28 \mathrm{ml} / \mathrm{min}$ approximately the same standard deviation. Thus, the reference (variant $R$ ) is comparable to conventional maximum permissible air leakage rates of $0.5-12 \mathrm{ml} / \mathrm{min}$ [4], not reliable to be watertight for the most demanding requirements. The observed leakage positions of variant $\mathrm{R}$ and the other variants are illustrated in Figure $7 \mathrm{~B}$. It is noticeable that air bubbles were emitted over the entire circumference of the metal insert, with the exception of the corners and the middle sections of the front faces of the metal insert.

In contrast to the leakage rate of variant $\mathrm{R}$, all other variants can also fulfill the most demanding requirements (Figure 7A). In addition, the different test methods show almost equal results, which is why they are not discussed separately in the following. The best results can be achieved with the microstructured variant MS, where no leakage rate could be measured with the used leakage tests. Considering the measurement resolution, the leakage rate of variant MS has to be less than $0.005 \mathrm{ml} / \mathrm{min}$ and therefore beyond measuring resolution. Furthermore, only at one test specimen, a single and tiny air bubble was observed at the middle section on one side of the metal insert (Figure 7B). The variants 0-100, I0-100 and I0-300 also yielded very good results with low leakage rates of approximately $0.01 \pm 0.01 \mathrm{ml} / \mathrm{min}$. Only variant $0-300$, with a structure depth of $0.3 \mathrm{~mm}$, shows a higher leakage rate compared to variant $0-100$, with a structure depth of $0.1 \mathrm{~mm}$. In all four embossed variants, the leakages were concentrated at the corner sections of the front faces of the metal insert. However, no air bubbles were observed at the corners of the metal inserts.

Based on the results of the leakage tests and the observation of the leakage positions, the following conclusions can be drawn for the embossed structures. First, all variants with embossed structures have a positive effect on the leakage rate compared to the untreated reference. Since variants I0-100 and I0-300, with lateral indentations, show similar leakage rates, it can be concluded that the structure depth has no relevant impact. Furthermore,
A

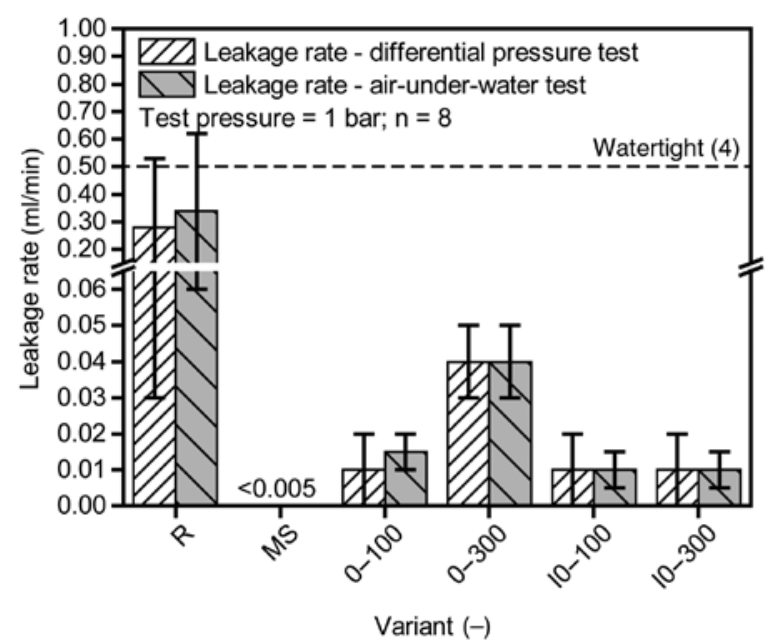

B

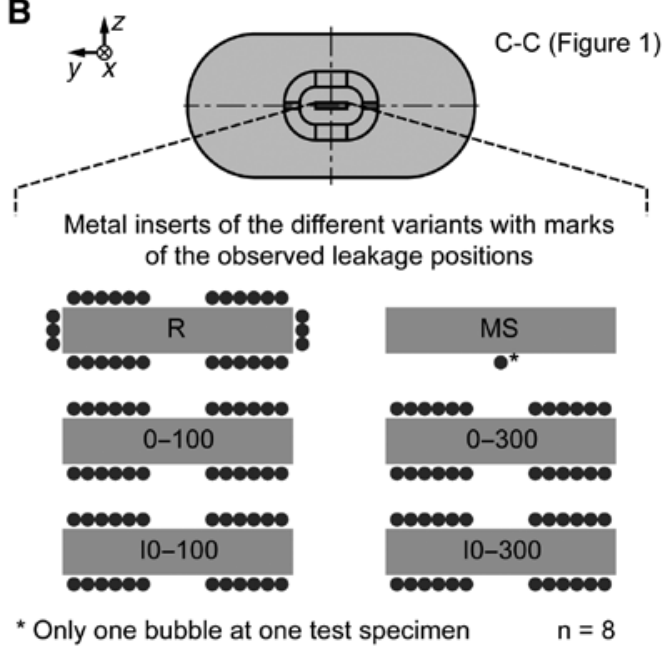

Figure 7: Leakage rates, measured with both differential pressure and air-under-water test (A) and the observed leakage positions (B) during the air-under-water test of all the variants. 
the design of the additional leakage barriers at the side faces has an influence on the leakage rate, but only in a small range (Figure $7 \mathrm{~A}$ ), variants $0-100,0-300, \mathrm{I0}-100$ and I0-300. Here, the distinct material bulges of variant 0-300 seem to have a negative effect on the leakage rate. The reasons for these effects are explained by the microscopy results in the next chapter.

\subsection{Microscopy}

In Figure $8 \mathrm{~B}$ and $\mathrm{D}$, the characteristic gap sizes of all variants are shown for all positions for the head (B) and bottom (D) sides in the $y z$ plane. Only for variant MS were no gaps measured, which is in accordance with the good results during the leakage test and corresponds to [18], where the formation of no gaps is explained by the polymer-filled microstructures with undercuts perpendicular to the metal surface. Furthermore, it can be seen that the gap profiles of variant $\mathrm{R}$ and those with embossed structures, which are respectively built by the characteristic gap sizes, are very similar. This is valid for both the head and bottom sides. Based on these gap profiles, especially at the bottom side, four representative sections can be defined: three for the front faces and one for the side faces of the metal insert (Figure 8B and D). The average values

A
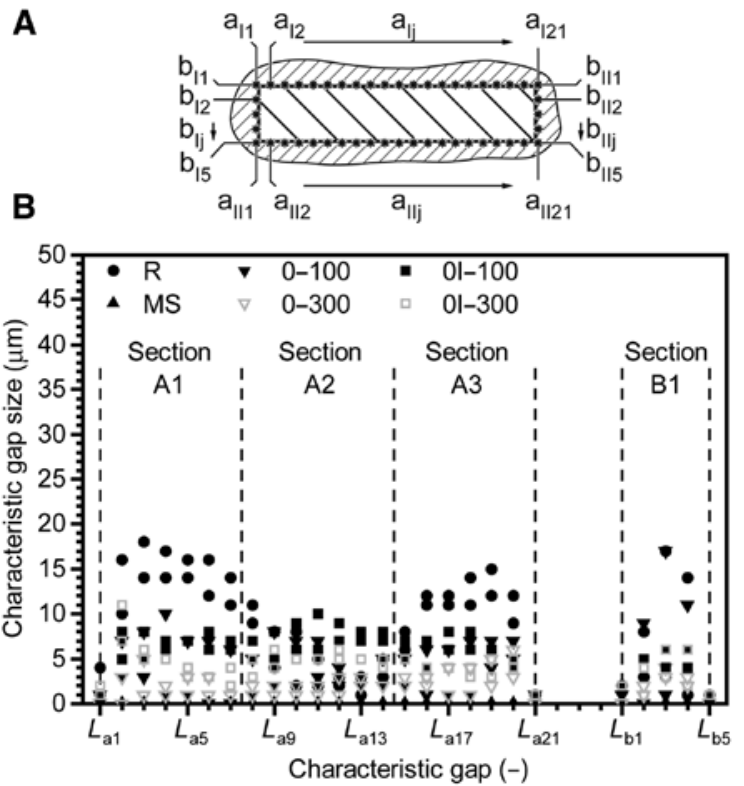

C

D
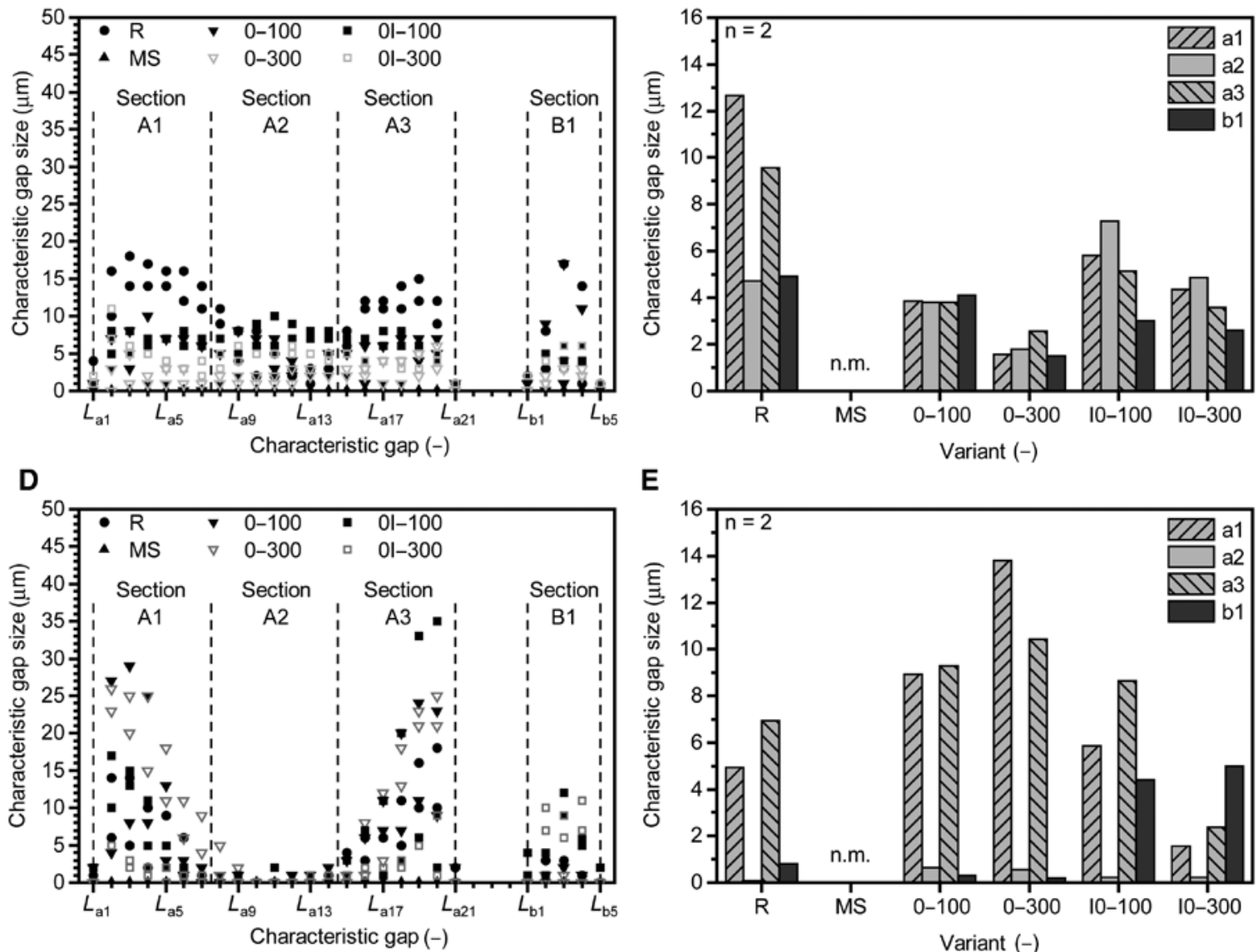

E

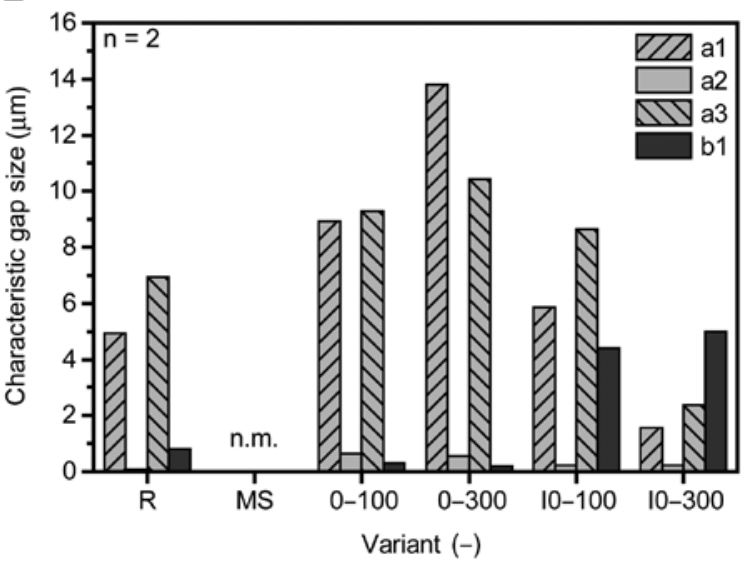

Figure 8: Characteristic gap sizes and average values $a 1, a 2, a 3$ and b1 of the respective characteristic gap sizes of the representative sections $A 1, A 2, A 3$ and $B 1$ in the $y z$ plane. (A) Measuring positions for the $y z$ plane as orientation. (B) Characteristic gap sizes of two test specimens for the head side and definition of the representative sections. (C) Average values of the characteristic gap sizes of $L_{\text {aj }}$ and $L_{\mathrm{bj}}$ for the representative sections of the head side. n.m. denotes that no gap could be measured. (D) Characteristic gap sizes of two test specimens for the bottom side and definition of the representative sections. (E) Average values of the characteristic gap sizes of $L_{\mathrm{aj}}$ and $L_{\mathrm{bj}}$ for the representative sections of the bottom side. n.m. denotes that no gap could be measured. 
a1, a2, a3 and b1 of the respective characteristic gap sizes of the representative sections $\mathrm{A} 1, \mathrm{~A} 2, \mathrm{~A} 3$ and $\mathrm{B} 1$ are shown in Figure $8 \mathrm{C}$ and $\mathrm{E}$, which facilitate the comparison of the variants.

At the head side, gaps can be measured at each position for variant $\mathrm{R}$ and for those with embossed structures between $L_{\mathrm{a} 1}$ and $L_{\mathrm{a} 21}$ as well as $L_{\mathrm{b} 1}$ and $L_{\mathrm{b} 5}$ (Figure 8B). At $L_{\mathrm{a} 1}$ and $L_{\mathrm{a} 21}$ as well as $L_{\mathrm{b} 1}$ and $L_{\mathrm{b} 5}$, which represent the corner areas of the metal insert, only very small or no gaps could be measured. The formation of these gaps at the front faces and side faces of the metal insert can be explained by the thermal contraction and crystalline shrinkage. At the corners of the metal insert, the thermal contraction of the polymer component, which is bigger than the one of the metal insert, leads to the closure of potential gaps during the cooling down while the predominant part of the polymer is still molten. Parallel to this, the crystalline shrinkage of the polymer, the base of which is a contour of the test specimen between the injection mold and the metal insert, leads to a gap formation between the corners. On closer consideration of variant $\mathrm{R}$ and those with embossed structures, a1-a3 are similar except for variant $R$, where a1 and a3 are significantly larger than a2 (Figure 8C). This difference can be explained by the embossed structures, which also influence the gap formation in areas without a structure. The explanation is supported by the comparison of the variants with embossed structures among themselves. Two trends can be identified here. First, the deeper the structure, the smaller the average values of the characteristic gap sizes. The only exception is b1 for variant I0-100/I0-300, which can be explained by the dominant indentations at the side faces, which may conceal effects of the material bulges. Second, through additional indentations at the side faces, the average values of the characteristic gaps increase at each representative section, with the largest increase located at section $\mathrm{A} 2$.

In contrast to the head side, gaps cannot be measured at each position for variant $R$ and those with embossed structures between $L_{\mathrm{a} 1}$ and $L_{\mathrm{a} 21}$ as well as $L_{\mathrm{b} 1}$ and $L_{\mathrm{b} 5}$ at the bottom side (Figure 8D). However, at the head side, only very small or no gaps could be measured in the corner areas. The reason for the gap formation at the corners is already explained for the head side. On closer consideration of variant $\mathrm{R}$ and those with embossed structures, it is conspicuous that the gap profiles and, thus, the average values of the characteristic gap sizes of the representative sections are significantly different to the ones of the head side, especially the differences between a1/a3 and a2. The reason why a1 and a3 are greater than a2 for all variants with the exception of variant MS is not yet clear. One theory is that the metal insert is very warm over an extended period of time, because the contact area of the metal insert with the injection mold is, due to its design, very small. Furthermore, the mold wall at the end of the flow path is $4 \mathrm{~mm}$ small and not actively cooled. These conditions in combination with the high thermal mass of the polymer and the high temperature of the injection mold lead to a slow cooling down of the polymer, especially in the vicinity of the corners of the metal insert. The result is a high crystallization degree at the corner areas, which results in a higher crystalline shrinkage at these positions. In addition, differences of the spherulite size between the corner areas and the areas between the corners may also have an influence on the crystalline shrinkage. Because of the long distance to the gate, this crystalline shrinkage presumably cannot be compensated by the holding pressure period. Furthermore, the local fiber orientations may also have an influence. The potential higher crystalline shrinkage in combination with the thermal contraction also leads to a higher overall shrinkage, which may be responsible for the small values of b1 with the exception of variants I0100 and I0-300, where the additional indentations also have a big influence. The influences of the crystallization degree, differences of the spherulite size and fiber orientation on the formation of gaps and the gap profile will be investigated in detail in further studies.

When comparing the average values of the characteristic gap sizes of the representative sections of the bottom side with the head side of variant $R$, a significant decrease in the average values can be seen for all sections (Figure $8 \mathrm{C}$ and $\mathrm{E}$ ). This is in line with the expectations, because the polymer dimensions around the metal insert are significantly smaller than those at the head side with its flange (Figure 2), and thus, the effects of the crystalline shrinkage on the gap formation are lower. However, when comparing a1, a2, a3 and b1 of the bottom side with those of the head side of the variants with embossed structures, no clear trend can be seen (Figure $8 \mathrm{C}$ and $\mathrm{E}$ ). In addition, the comparison of the average values of the characteristic gap sizes of the representative sections at the bottom side (Figure 8E) only shows that the additional indentations have a big influence on the gap formation at section B1. These results show that there must be a complex relationship between the structures and shrinkage, which cannot be seen at the head side, where the flange prevents dislocations of the polymer on the crosspiece of the metal insert caused by shrinkage. However, the average values of the characteristic gap sizes of the representative sections of all variants are in good accordance with the observed leakage positions. Only in variants I0-100 and I0-300 were no air bubbles observed at the side faces, although large gaps 
were measured. The reason for this is analyzed in the $x y$ and $x z$ planes in the following. Furthermore, the average values of the characteristic gap sizes of the representative sections of variants R and MS are also in good accordance with the observed (R) or non-existent (MS; only at one test specimen a single air bubble was seen) leakage positions.

The characteristic gap sizes of the $x z$ plane are shown in Figure 9 for the head (C) and bottom sides (E) of all variants with embossed structures. It can be seen that at the head side, all variants have gaps at each position of the embossed structure, so that no leakage barrier is generated. The main reason for this is the position of the structure, which is located in the area of the flange of the polymer component. At this position, the flange prevents a dislocation of the polymer component in the direction of the crosspiece of the metal insert, which is required for the leakage barrier. Thus, the polymer shrinks only perpendicular to the metal surface in the direction of the polymer base as described before, which leads to a gap formation at the structure ground, represented by $L_{\mathrm{d}}$. However, because the structure flanks are not perpendicular to the structure ground, gaps also formed at these positions, represented by $L_{\mathrm{c}}$ and $L_{\mathrm{e}}$. At the bottom side, where a dislocation of the polymer is not prevented, the $L_{\mathrm{c}}$ of all the variants is very small or cannot be measured. Furthermore, the shrinkage of the polymer in the direction of the flange leads to an opening of $L_{\mathrm{e}}$, when comparing the proportion of $L_{\mathrm{d}}$ to $L_{\mathrm{e}}$ at the head and bottom sides. When comparing $L_{\mathrm{d}}$ of the bottom and head sides of all variants, a significant decrease in the values can be seen. This can be explained by the crystalline shrinkage perpendicular to the metal surface in the direction of the
A
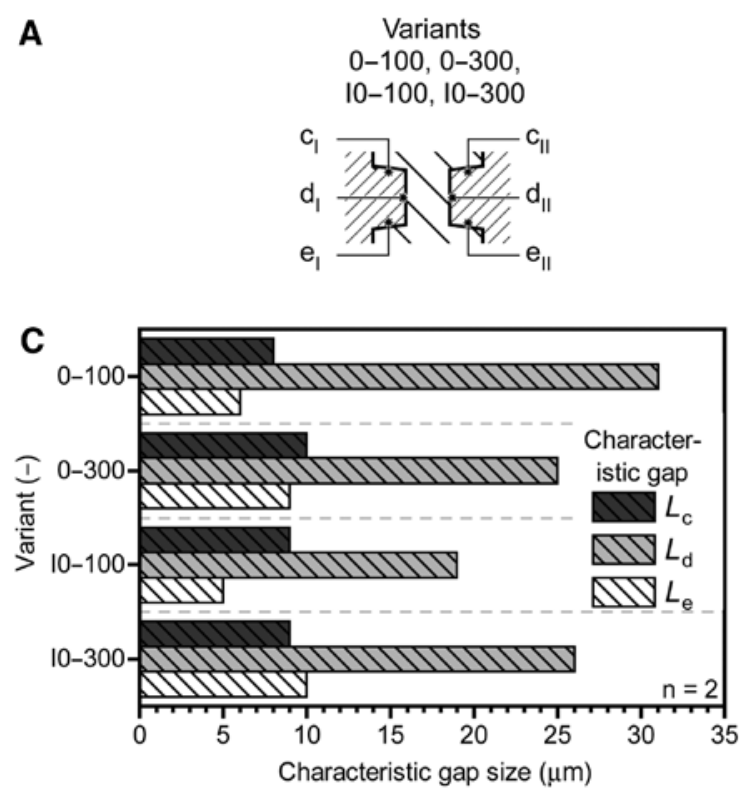

E

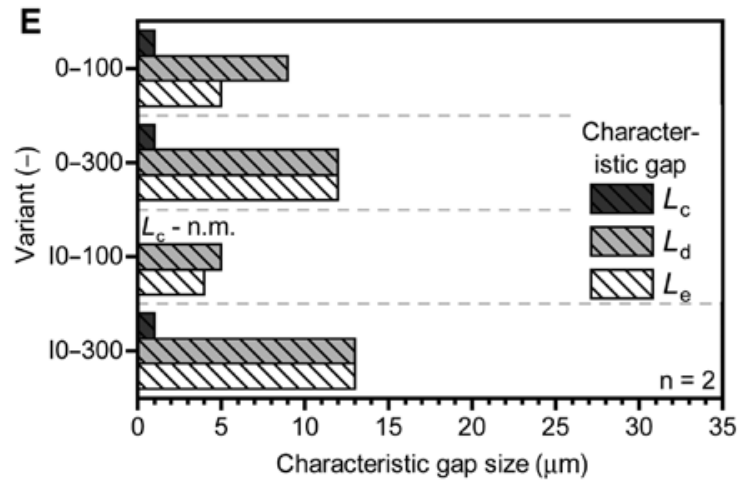

B
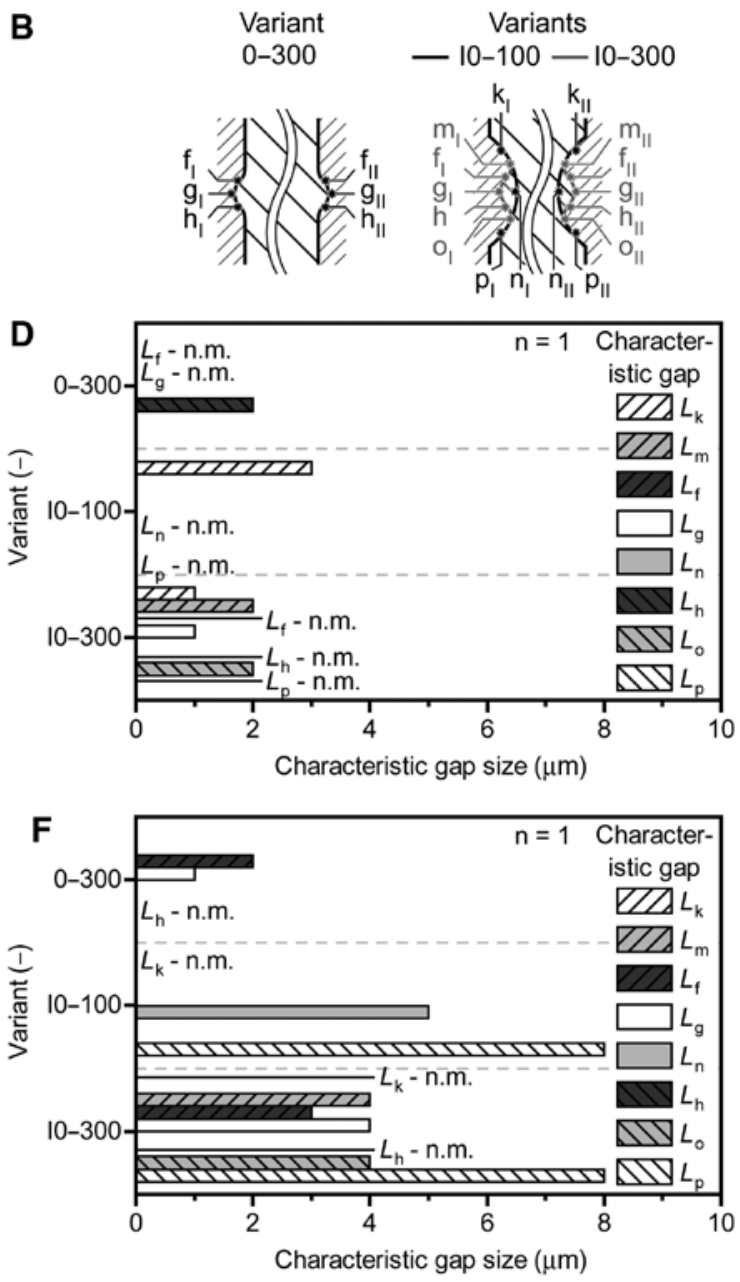

Figure 9: Characteristic gap sizes in the $x y$ and $x z$ planes for the head and bottom sides. n.m. denotes that no gap could be measured. (A) Measuring positions for the $x z$ plane as orientation. (B) Measuring positions for the $x y$ plane as orientation (C) Characteristic gap sizes in the $x z$ plane for the head side. (D) Characteristic gap sizes in the $x y$ plane for the head side. (E) Characteristic gap sizes in the $x z$ plane for the bottom side. (F) Characteristic gap sizes in the $x y$ plane for the bottom side. 
polymer base, because the polymer dimensions around the metal insert at this position are significantly smaller than those at the head side with its flange (Figure 2). The measured characteristic gap sizes are in good accordance with the measured leakage rates of these variants. Two main conclusions can be drawn from this. First, structures across the crosspiece of the metal insert, which should be used as a leakage barrier, must be deeper than $L_{\mathrm{d}}$ in the $x z$ plane plus the maximum of $L_{\mathrm{aj}}$ in the $y z$ plane. Second, these structures have to be placed at areas in which the shrinkage is not hindered by the injection mold and which have a maximum distance to the center of the shrinkage.

The characteristic gap sizes of the $x y$ plane are shown in Figure 9 for the head (D) and bottom sides (F). At the head and bottom sides of variant $0-300, L_{\mathrm{g}}$ is not measurable or measures only $1 \mu \mathrm{m}$. This is in rough accordance with the small values of b1 in the $y z$ plane (Figure 8C and D), which describes the gaps at similar positions at the side faces of the metal insert, but outside of the structured areas. For the other characteristic gaps, $L_{\mathrm{h}}$ can only be measured at the head side and $L_{\mathrm{f}}$ only at the bottom side, which is in line with the expectations of the shrinkage behavior. However, all characteristic gaps at both sides are very small or not measurable, which is in contrast to the characteristic gaps in the $x z$ plane with similar geometrical conditions. The only main difference between these two planes is the considered dimension of the metal insert, $1 \mathrm{~mm}$ in the $z$ direction ( $x z$ plane) and $5 \mathrm{~mm}$ in the $y$ direction ( $x y$ plane). Thus, it can be concluded that the dimensions of the metal insert in combination with the thermal contraction probably are responsible for the differences of the sizes of the characteristic gaps. However, even if the characteristic gaps are very small in the $x y$ plane, they give a clue about the gap formation at the flanks of the material bulges.

For variant I0-100, the $L_{\mathrm{n}}$ of the head and bottom sides shows the same trends as the $L_{\mathrm{g}}$ at variant $0-300$, but the difference is more distinct. The reason for this may be the indentations of the metal insert at the bottom side, which act as wedges during the shrinkage of the polymer in the $x$ direction and, thus, open small gaps. In addition, the high internal stress in the polymer, which would be a result of the hindered shrinkage, could explain the value of b1 at the bottom side (Figure 8E), where a complex relationship between the structures and the shrinkage is already inferred. The characteristic gap size $L_{\mathrm{k}}$ at the bottom side, which is not measurable in contrast to $L_{\mathrm{p}}$, supports this argumentation. However, in contrast to $L_{\mathrm{d}}$ and $L_{\mathrm{e}}$ of the embossed structures in the $x z$ plane, which describe similar geometrical conditions, $L_{\mathrm{n}}$ and $L_{\mathrm{p}}$ are not measurable at the head side. These differences can be explained analogous to variant $0-300$ by the dimensions of the metal insert in combination with the thermal contraction.

For variant I0-300, $L_{\mathrm{g}}, L_{\mathrm{m}}$ and $L_{\mathrm{o}}$ of the head and bottom sides show the same trends like $L_{\mathrm{g}}$ and $L_{\mathrm{n}}$ at variants $0-300$ and I0-100. Analogous to variant I0-100, the reason for this may be the indentations of the metal insert at the bottom side. As mentioned for variant I0-100, the high internal stress in the polymer as a result of the hindered shrinkage could explain the value of b1 at the bottom side (Figure 8E). Furthermore, in contrast to $L_{\mathrm{f}}$ and $L_{\mathrm{o}}, L_{\mathrm{k}}$ and $L_{\mathrm{h}}$ are not measurable at the bottom side, which is in accordance with the gap formation of variant I0-100 and, thus, supports this argumentation. However, as for variant I0-100, no or only small values can be measured for the characteristic gaps at the head side. The probable explanation is the same, which is already given for variant $0-300$

When evaluating the characteristic gaps in the $x y$ plane, it must be noted that only one test specimen per variant was analyzed, and thus, the results only show trends. Nevertheless, the results are in good accordance with the observed leakage positions. Especially, when looking at Figure 8E, the results of variants I0-100 and I0-300 in Figure 9F show why no air bubbles could be observed at the side faces (Figure 7B). Furthermore, based on the measured leakage rates and the characteristic gaps in the $x y$ and $x z$ planes, it can be concluded that the design of the structures has an effect on the leakage rate. If the structures at the front faces and side faces are designed differently, for example, through material removal at the front faces and application of material on the side faces, the leakage rate is increased. The reason for this is that the leakage barrier is not closed, because the edges on which the gaps are closed due to the shrinkage of the polymer are different, for example, variant 0-300 (Figure 9E and F). If the structures at the front faces and side faces are designed in the same way, for example, through material removal, the leakage rate is constantly low. In order to analyze the interactions between the front faces and the side faces in detail, further investigations with more test specimens are necessary. Furthermore, metal inserts with other ratios of the side faces to the front face are needed. In addition, for the analysis of the effects shown for variant 0-300, larger structures should be used.

In summary, the microscopy results show where the gaps are formed at the different variants at the head and bottom sides in the $x y, x z$ and $y z$ planes. Through the combination of these results, the formation of leakage networks can be explained for the investigated variants. At variant $R$, gaps at the side faces and front faces run from the head side to the bottom side, forming a leakage 
network without any leakage barriers (Figure 10A). In contrast, no gaps could be measured at variant MS, where the polymer-filled microstructures prevent any gap formation in the different planes. For the variants with embossed structures, the leakage networks are more complicated. In the range of the polymer flange, structures at the front faces cannot form leakage barriers and only extend the leakage path. However, at the bottom side, structures at the front faces straighten the leakage paths but cannot completely avoid the formation of a leakage network. At the side faces, structures can prevent the formation of leakage paths. However, only at one structure can flank gaps be closed (Figure 9D and F). Hence, if the structures are designed unfavorably, they can interact with those of the front faces, because there are also very small gaps at the edges of the metal insert (Figure 10B). If the structures at the side faces are designed favorably, which means that they are designed in the same way as the structures at the front faces, they can form, in combination with those of the front faces, a closed leakage barrier around the metal insert (Figure 10C). The fact why no air bubbles could be observed at the side faces during the air-under-water test of all variants with embossed structures can be explained by the interaction of the gaps behind the last structure at the bottom side (in the test direction). At variants 0-300, I0-100 and I0-300, there is only a small leakage path at
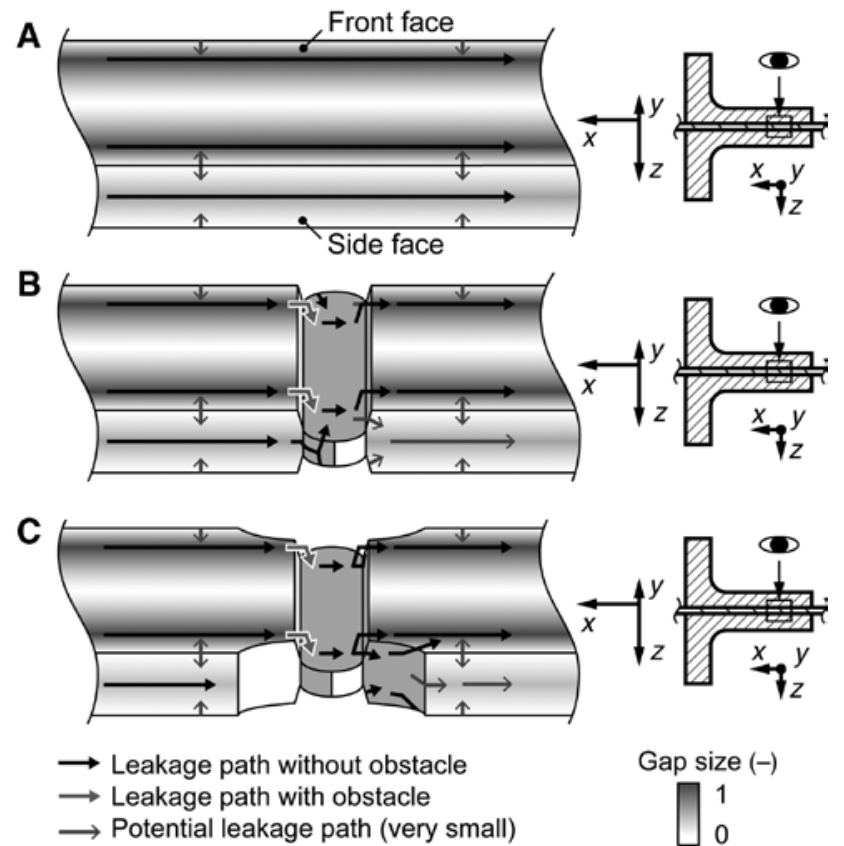

Figure 10: Formation of the leakage network of variants $R(A)$, 0-300 (B) and 10-300 (C) on the surface of the metal insert at the bottom side of the test specimen. The material bulges and structure flanks are illustrated disproportional. the front faces (Figure 9E and F). In addition, the gaps after the last structure are larger than those in the structure, and the air pressure is significantly reduced, so that the air flows through the gaps on the front faces out of the test specimen. The very small gaps at the corners of the metal insert in combination with the reduced air pressure and the small gaps at the side faces of the metal insert seem to be too small to form leakage paths under the given conditions but are also potential leakage paths. For variant $0-100$, the gaps at the side faces and the corners of the metal insert are also very small, so that potential leakage paths may be closed completely at a minimum of one position or they lead to the front faces of the metal insert.

\section{Conclusion and outlook}

In the present work, the formation of gap-based leakages in polymer-metal electronic systems with labyrinth seals has been investigated using a polymer-metal connector test specimen with a rectangular metal insert (aspect ratio 5:1). In total, five variants of labyrinth seals on the metal insert were analyzed: four variants with embossed structures on the front faces, which differ by the depth of the structures and partially additional leakage barriers on the side faces of the metal insert, and one variant with an area seal manufactured by an electrochemical microstructuring. For the analysis of the tightness, a differential pressure test was used in combination with an air-under-water test, which in addition enables the location of leakage positions. The formation of the measured leakages and the mechanism of action of the used labyrinth seals were analyzed by cross sections of the bottom and head sides of the test specimen in three planes by microscopy.

The results of the leakage tests show that the investigated labyrinth seal variants enable a significant improvement of the tightness compared to a reference without labyrinth seal. The best result is achieved with the electrochemically manufactured area seal, where no leakage and gaps could be measured. For the other variants, the leakage-relevant gaps are identified and the leakage positions are located, which in total allows for derivation of the respective leakage network. Furthermore, the mechanism of action of the structures at the head and bottom sides of the test specimen is shown. In summary, four main conclusions can be drawn by these results, which are relevant for the component design:

(1) The suitable position of a structure, which should be used as a labyrinth seal, depends on the possible 
shrinkage of the polymer in the injection mold. In order to enable high tightness, the structures must be placed at areas of the metal insert, in which the shrinkage of the polymer is not hindered by the injection mold and which have an as-large-as-possible distance to the center of the shrinkage of the polymer.

(2) Structures for the labyrinth seals should be deeper than the gaps caused by the shrinkage of the polymer perpendicular to the metal surface.

(3) Labyrinth seals should be located around the whole metal insert and designed in the same way, for example, all around groove, to ensure good tightness.

(4) Microstructures with undercuts perpendicular to the surface of the metal insert are favorable for tight polymer-metal electronic systems, since the interlock between the metal and the polymer prevents the formation of gaps. However, according to [15], the basic requirement for this is that the microstructures are filled with polymer.

Further research will focus on the investigation of the influences of the crystallization degree, differences of the spherulite size and fiber orientation on the formation of gaps and their profile. Furthermore, the interaction between the surfaces of rectangular metal inserts with different aspect ratio of the side and front faces will be investigated in detail.

Acknowledgments: The authors thank the German Research Foundation (DFG) for funding this study within the project ME 2043/33-2 and DR 421/10-2, Funder Id: http://dx.doi.org/10.13039/501100001659, “Fundamental investigation of blanking, forming and assembly injection molding and their interdependence for manufacturing durable tight electronic systems."

\section{References}

[1] Schaefer U. Mikroelektronik - Trendanalyse bis 2021. Vorstellung langfristiger Trends 2011-2016-2021. Available at: https://www. zvei.org/fileadmin/user_upload/Presse_und_Medien/Publikationen/2017/Mai/Mikroelektronik_Trendanalyse_bis_2021/201705_Mikroelektronik-Trendanalyse-bis-2021.pdf (2017-11-14).

[2] Zabler E, Finkbeiner S, Welsch W, Kittel H, Bauer C, Noetzel G, Emmerich H, Hopf G, Konzelmann U, Wahl T, Neul R, Müller W, Bischoff C, Pfahler C, Weiberle P. In Sensoren im Kraftfahrzeug, 3rd ed., Reif K, Ed., Springer Vieweg: Wiesbaden, 2016, p 2-25.
[3] Osswald TA, Baur E, Brinkmann K, Oberbach K, Schmachtenberg E, Eds., International Plastics Handbook. The Resource for Plastics Engineers, 4th ed., Carl Hanser Verlag: Munich, 2006.

[4] Müller J. Leitfaden zur Industriellen Dichtheitsprüfung, Training material, Dr. Wiesner Steruerungstechnik GmbH, 2011.

[5] Heinle C, Vetter M, Ehrenstein GW, Drummer D. J. Manuf. Sci. Eng. 2010, 132, 054505-1-054505-4.

[6] Heinle C, Vetter M, Brocka-Krzeminska Z, Ehrenstein GW, Drummer D. J. Plast. Technol. 2009, 5, 428-450.

[7] Byskov-Nielsen J, Hiolm AH, Hojsholt R, Sá P, Balling P. Appl. Phys. A 2011, 104, 975-979.

[8] Grishin A, Knospe A, Buske C. Proc. Eurotech, Lyon (France) 2013, 2014, 187-192.

[9] Schulz T. Methods and materials to achieve tight metalpolymer-composites, 8th International Congress - Molded Interconnect Devices, Nuremberg-Fuert (Germany) 2008.

[10] Eltermann F, Wilde J. Proc. 3rd Symposium on Innovations in Adhesives and their Applications, Munic (Germany) 2017, 1, 51-63.

[11] Eltermann F, Wilde J. Proc. TechnoBond, Bad Hersfeld (Germany) 2017, 3, 126-129.

[12] Melamies IA. Kunststoffe Int. 2016, 2016, 8-13.

[13] Packham DE. In Theories of fundamentals adhesion, 1st ed., da Silva LFM, Öchsner A, Adams RD, Eds., Springer-Verlag Berlin Heidelberg: Heidelberg, 2011, 9-38.

[14] Lucchetta G, Marinello F, Bariani PF. CIRP Ann. Manuf. Techn. 2011, 60, 559-562.

[15] Grujicic M. Am. J. Sci. Technol. 2014, 1, 168-181.

[16] Zentgraf T. Mediendichtes Umspritzen von Einlegeteilen aus Metall und Keramik, research report (AIF project number16161 N), 2011.

[17] Bonpain B. Entwicklung und prozesstechnische Analyse eines festen und mediendichten Kunststoff - Metall - Verbundes, Lehrstuhl für Polymerwerkstoffe, Ed., Ph.D. thesis, Dortmund, 2017.

[18] Kleffel T, Drummer D. SPE Proc. ANTEC, Orlando (USA) 2018.

[19] Kleffel T, Drummer D. J. Polym. Eng. 2018, 28, 675-684.

[20] Kleffel T, Drummer D. Compos. Part B Eng. 2017, 117, 20-25.

[21] Heinle M, Frey F, Merklein M, Drummer D. J. Plast. Technol. 2018, 03, 212-232.

[22] Frey P, Heinle M, Drummer D, Merklein M. MATEC Web of Conferences 2015, 21, 43 09013-1-09013-7.

[23] Frey P, Heinle M, Leisen C, Drummer D, Merklein M. Key Eng. Mater. 2015, 639, 99-106.

[24] DIN 8583-5. Manufacturing Processes Forming Under Compressive Conditions - Part 5: Indentation-Forming; Classification, Subdivision, Terms and Definitions. Beuth, 2003.

[25] Geiger M, Kleiner M, Eckstein R, Tiesler N, Engel U. Ann. CIRP 2001, 2, 445-462.

[26] Thome M, Hirt G, Rattay B. Adv. Mater. Res. 2005, 6-8, 631-638.

[27] Stellin T, van Tijum R, Merklein M, Engel U. Key Eng. Mater. 2014, 611-612, 565-572.

[28] DIN EN ISO 291. Plastics - Standard Atmosphere for Conditioning and Testing. Beuth, 2008. 